\title{
Rompimento de barragens na literatura científica internacional: Uma análise
}

\section{bibliométrica}

\author{
Dam disruption in international scientific literature: A bibliometric analysis \\ Interrupción de presas en la literatura científica internacional: Un análisis bibliométrico
}

Recebido: 08/04/2021 | Revisado: 15/04/2021 |Aceito: 17/04/2021 | Publicado: 29/04/2021

\author{
Lázaro Avelino de Sousa \\ ORCID: https://orcid.org/0000-0001-6505-1288 \\ Universidade Federal de Campina Grande, Brasil \\ E-mail: lazaroavelino@hotmail.com \\ Sérgio Murilo Santos de Araújo \\ ORCID: https://orcid.org/0000-0001-9599-4383 \\ Universidade Federal de Campina Grande, Brasil \\ E-mail: sergiomurilosa.ufcg@gmail.com \\ Maria de Fátima Nóbrega Barbosa \\ ORCID: https://orcid.org/0000-0003-3415-8829 \\ Universidade Federal de Campina Grande, Brasil \\ E-mail: mfnobregabarbosa@gmail.com
}

\begin{abstract}
Resumo
O rompimento de barragens é um tema que recentemente ganhou destaque na mídia, sobretudo, devido a grandes desastres envolvendo perdas de vidas humanas. Uma análise bibliométrica do tema é oportuna para que se possa sistematizar estudos com embasamento científico para subsidiar medidas de segurança e prevesão deste tipo de acidente. Neste sentido, este trabalho analisou a produção científica internacional sobre rompimento de barragens através de uma abordagem quantitativa que objetivou mensurar a dinâmica bibliométrica do tema no período compreendido entre os anos de 1945 e início de 2021. Foi feita uma pesquisa na coleção principal da base de dados interdisciplinar Web of Science considerando-se como principais critérios o recorte temporal supracitado e a ocorrência da palavra-chave "Dam Break" no título das publicações. Pesquisou-se apenas pela modalidade artigo, publicado na língua inglesa. Os metadados da pesquisa foram baixados no formato de planilha do Excel e foram aplicadas estatísticas descritivas para classificar e quantificar as informações. Os resultados mostram que a ocorrência do tema na literatura internacional só se verifica a partir do ano de 1980, tendo como principal veículo de comunicação o Journal of Hydraulic Research, e o principal país de origem, a China. O total de publicações encontradas foi de 306 artigos, sendo 44 considerados de maior impacto na literatura científica, de acordo com o Hindex. Conclui-se que o tema pesquisado vem crescendo em importância nos últimos anos com o aumento do número de publicações, principalmente nas áreas de Recursos Hídricos e Engenharia Civil, a partir do ano de 2006.
\end{abstract}

Palavras-chave: Rompimento de barragens; Web of Science; Bibliometria.

\begin{abstract}
The breaking of dams is a topic that has recently gained prominence in the media, mainly due to major disasters involving loss of human lives. A bibliometric analysis of the topic is opportune so that studies with a scientific basis can be systematized to support safety measures and prevention of this type of accident. In this sense, this work analyzed the international scientific production on the rupture of dams through a quantitative approach that aimed to measure the bibliometric dynamics of the theme in the period between the years 1945 and the beginning of 2021. A survey was carried out in the main collection of the database. interdisciplinary data Web of Science considering as main criteria the aforementioned time frame and the occurrence of the keyword "Dam Break" in the title of the publications. We only searched for the article modality, published in the English language. The survey metadata was downloaded in Excel spreadsheet format and descriptive statistics were applied to classify and quantify the information. The results show that the occurrence of the theme in the international literature only occurs from the year 1980, with the Journal of Hydraulic Research as the main vehicle of communication, and the main country of origin, China. The total number of publications found was 306 articles, 44 of which were considered to have the greatest impact in the scientific literature, according to the H-index. It is concluded that the researched theme has been growing in importance in recent years with the increase in the number of publications, mainly in the areas of Water Resources and Civil Engineering, since 2006.
\end{abstract}

Keywords: Dam break; Web of Science; Bibliometry. 


\section{Resumen}

La ruptura de represas es un tema que recientemente ha ganado protagonismo en los medios de comunicación, principalmente debido a los grandes desastres que implican la pérdida de vidas humanas. Un análisis bibliométrico de la temática es oportuno para que se puedan sistematizar estudios con base científica que apoyen las medidas de seguridad y prevención de este tipo de accidentes. En este sentido, este trabajo analizó la producción científica internacional sobre la ruptura de presas a través de un enfoque cuantitativo que tuvo como objetivo medir la dinámica bibliométrica del tema en el período comprendido entre los años 1945 y principios de 2021. Se realizó una encuesta en el principal colección de la base de datos datos interdisciplinarios Web of Science considerando como criterio principal el marco temporal antes mencionado y la ocurrencia de la palabra clave "Dam Break" en el título de las publicaciones. Solo se buscó la modalidad de artículo, publicado en idioma inglés. Los metadatos de la encuesta se descargaron en formato de hoja de cálculo Excel y se aplicaron estadísticas descriptivas para clasificar y cuantificar la información. Los resultados muestran que la ocurrencia del tema en la literatura internacional solo ocurre a partir del año 1980, con la Revista de Investigaciones Hidráulicas como principal vehículo de comunicación, y el principal país de origen, China. El número total de publicaciones encontradas fue de 306 artículos, 44 de los cuales se consideraron de mayor impacto en la literatura científica, según el H-index. Se concluye que el tema investigado ha ido cobrando importancia en los últimos años con el incremento en el número de publicaciones, principalmente en las áreas de Recursos Hídricos e Ingeniería Civil, desde 2006.

Palabras clave: Ruptura de presa; Web of Science; Bibliometría.

\section{Introdução}

A técnica de construção de barragens tem séculos de aperfeiçoamento em função do tipo de uso e da experiência prática adquirida com os estudos de rompimento dessas estruturas. Pode-se considerar que acidentes associados ao rompimento de barragens vêm sendo observados desde que barragens passaram a ser construídas de forma sistemática para seus mais diversos propósitos (Tschiedel et al., 2019). De acordo com a International Commission on Large Dams (2018), 59 mil barramentos com mais de 15 metros de altura e mais de $3 \mathrm{hm}^{3}$ de volume estão distribuídos pelo mundo, tendo como principais finalidades a irrigação, a geração de energia elétrica e o abastecimento populacional. Estes três setores respondem por $80 \%$ das barragens destinadas para um único fim.

Diante deste volume de barramentos e do risco potencial associado que eles representam em termos de acidente, Tschiedel (op. cit.), baseado em estudos de Zhang et al. (2016) e Mao et al. (2017), estima que há aproximadamente um rompimento de barragem a cada 4 dias no mundo, considerando-se em escala global o período de 1954 a 2006. A perda de vidas humanas associadas a este tipo de tragédia apresenta números elevados. De acordo com Proske (2018), o número de pessoas mortas por rompimento de barragens no século 20, excluindo a China, é estimado em aproximadamente 13.500 vítimas fatais.

No cenário internacional alguns casos emblemáticos de acidentes com rompimento de barragens podem ser destacados, como o caso da St. Francis Dam, na Califórnia-EUA, ocorrido em 12 de março de 1928. Com problemas nas ombreiras, a barragem vitimou fatalmente 460 pessoas e destruiu dez pontes e 1.200 casas. Outro caso famoso ocorreu na Itália em $1^{\circ}$ de outubro de 1963, quando o talude rochoso da barragem de Vajont rompeu e caiu sobre o reservatório a $50 \mathrm{~m}$ da crista da barragem. A onda sobre a crista destruiu a cidade de Longarone e vitimou fatalmente 1.925 pessoas. Na china, em 07 de agosto de 1975, após chuvas com período de retorno de 2 mil anos, 240 mil pessoas morreram e cerca de 2 milhões ficaram desabrigadas após o rompimento de duas barragens principais (Banqiao e Shimantan Henan) e outras 62 em seguida (Spragens \& Mayfield, 2005; Muller-Salzburg, 1987; Panizzo et al., 2005; Mccully, 1999; Pisaniello, Zhifang \& Mckay, 2006, como citado em Sánchez, 2013, p. 362).

No Brasil, os casos mais emblemáticos são os recentes desastres de Mariana e Brumadinho ocorridos em Minas Gerais nos anos de 2015 e 2019. Ambos os casos foram registrados em barragens de rejeitos de mineração. No caso de Mariana, a barragem de Fundão rompeu no dia 05 de novembro de 2015, despejando 34 milhões de metros cúbicos de lama na bacia do rio Doce (Agência Nacional de Águas, 2016).

De acordo com Castro e Almeida (2019), os rejeitos e detritos liberados pelo rompimento da barragem de Fundão 
seguiram os cursos do córrego Santarém e dos rios Gualaxo do Norte e do Carmo por cerca de 77 km, até alcançar o rio Doce. Ao todo, a lama percorreu uma extensão de 663,2 km até a foz do Rio Doce no oceano Atlântico, provocando danos em 41 municípios com a suspensão imediata do abastecimento de água, da atividade pesqueira e do turismo na região. De acordo com Laschefski (2020), o desastre de Mariana causou 19 mortes e deixou cerca de 1.200 famílias desabrigadas.

No caso de Brumadinho, no dia 25 de janeiro de 2019 a barragem de rejeitos B1 da mina de Córrego do Feijão, de propriedade da empresa Vale S.A., rompeu-se, soterrando mais de 270 pessoas e despejando 13 milhões de $\mathrm{m}^{3}$ de rejeitos úmidos, produzidos pela extração de minério de ferro, no leito do ribeirão Ferro-Carvão (Neves-Silva \& Heller, 2020). De acordo com a Fundação Oswaldo Cruz (2019), a onda de lama alastrou-se pela comunidade de Córrego do Feijão e chegou a Brumadinho, atingindo várias cidades ao longo do rio Paraopeba. O desastre destruiu casas, afetou plantações e zonas de criação de animais, além de poluir o rio e dificultar o acesso à água em várias comunidades.

De acordo com Ferla (2018), as principais causas já observadas no rompimento de barragens de todos os tipos são as causas de galgamento, erosão interna e falhas nas fundações. Além disso, segundo a autora, outras causas podem ser destacadas, como as falhas de projeto, construção e operação, sismos, terremotos e ações de guerra.

A temática do rompimento de barragens tem crescido em importância ao longo dos anos na literatura acadêmica com o aperfeiçoamento das técnicas de construção que possibilitaram o aumento da capacidade de acumulação desses reservatórios e a diversificação de seu uso, como abastecimento de água, geração de energia, disposição de rejeitos, entre outras.

Nos Estados Unidos, desde o final dos anos 1970, já haviam sido desenvolvidos os primeiros estudos de risco de barragens, impulsionados pela ruptura de uma barragem no Estado da Georgia (Spragens \& Mayfield, 2005).

No Brasil, a legislação sobre segurança de barragens data do ano de 2010 . Trata-se da lei federal $\mathrm{n}^{\circ} 12.334$, de 20 de setembro de 2010, que "estabelece a Política Nacional de Segurança de Barragens destinadas à acumulação de água para quaisquer usos, à disposição final ou temporária de rejeitos e à acumulação de resíduos industriais, e cria o Sistema Nacional de Informações sobre Segurança de Barragens" (Lei nº 12.334, 2010).

Em escala estadual, o Estado de Minas Gerais, através da Fundação Estadual do Meio Ambiente (Feam), depois de diversos eventos de rupturas de barragens de rejeitos de minas e industriais, obrigou todas as empresas responsáveis por barragens a fazer cadastro, o primeiro passo para um programa público de gerenciamento de riscos (Torquetti \& Farias, 2004).

No Brasil, o Movimento dos Atingidos por Barragens (MAB) existe desde o ano de 1980, e organiza os atingidos por barragens, antes, durante e depois da construção dos empreendimentos. De acordo com o site do Movimento na internet (https://mab.org.br/), o Relatório de Segurança de Barragens de 2018 confirma que existem no Brasil 17.604 barragens cadastradas nos 32 órgãos fiscalizadores (municipais, estaduais e nacional). Deste total, 5.086 foram classificadas por Categoria de Risco e 6.577 por Dano Potencial Associado, sendo que 909 estão classificadas simultaneamente como Categoria de Risco e Dano Potencial Associado altos.

Nesse contexto, a abordagem do tema rompimento de barragens na atualidade é de fundamental importância, tanto para a segurança hídrica, quanto para a prevenção de desastres. É de interesse para os empreendedores do ramo de produção de energia, de mineração, pesca, e, sobretudo, para as populações residentes à jusante desses empreendimentos.

Reconhecendo a importância do tema, este trabalho analisou a produção científica internacional sobre rompimento de barragens através de uma abordagem quantitativa que objetivou mensurar a dinâmica bibliométrica do tema no período compreendido entre os anos de 1945 e início de 2021, através de pesquisa na rede mundial de computadores.

\section{Metodologia}

A pesquisa da bibliografia sobre o tema em tela foi feita na coleção principal da base de dados interdisciplinar Web of Science, uma plataforma referencial de citações científicas projetada para apoiar pesquisas científicas e acadêmicas com 
cobertura nas áreas de ciências, ciências sociais, artes e humanidades. A coleção principal da Web of Science inclui mais de 20.000 revistas acadêmicas de alta qualidade revisadas por pares e publicadas em todo o mundo, e mais de 190.000 processos de conferências (Universidade de Brasília, 2018).

A pesquisa dos artigos na base Web of Science foi feita a partir da plataforma de acesso remoto denominada Comunidade Acadêmica Federada (CAFE), acessada a partir do Portal de Periódicos da Coordenação de Aperfeiçoamento de Pessoal de Nível Superior (CAPES). Como critério principal utilizou-se o termo "Dam Break" como palavra-chave contida no título das publicações. Pesquisou-se por todos os anos (de 1945 a 2021), utilizando-se os índices SCI-EXPANDED, SSCI e ESCI na coleção principal da Web of Science. A pesquisa e geração do banco de dados foi feita no dia 15 de fevereiro 2021 , às 15h:25min.

Para a triagem das publicações de interesse relativas ao tema pesquisado fez-se o refinamento por tipo de documento e por categorias da própria base Web of Science. Pesquisou-se por "ARTICLE” nas categorias "WATER RESOURCES" ou “ENGINEERING CIVIL” ou "GEOSCIENCES MULTIDISCIPLINARY” ou “ENVIRONMENTAL SCIENCES” ou “METEOROLOGY ATMOSPHERIC SCIENCES”. Com tais critérios obteve-se um total de 323 artigos.

Do total de artigos encontrados, eliminou-se os artigos de prelo utilizando-se o filtro "PROCEEDINGS PAPER OR EARLY ACCESS” e obteve-se um total de 313 artigos. Em cima deste último resultado aplicou-se mais dois filtros: por idioma "ENGLISH”, e por áreas de conhecimento: "WATER RESOURCES” ou "ENGINEERING” ou "PHYSICAL GEOGRAPHY" ou "GEOLOGY" ou "ENVIRONMENTAL SCIENCES ECOLOGY" ou "URBAN STUDIES" ou “METEOROLOGY ATMOSPHERIC SCIENCES”. Os resultados dos dois últimos filtros mostraram um resultado igual a 306 artigos publicados entre os anos de 1980 e 2021, sendo este o resultado final da triagem.

Assim, a base de dados utilizada neste estudo consiste em 306 artigos científicos, publicados em inglês, em periódicos indexados na coleção principal da base Web of Science, dentro das áreas de estudo "Recursos Hídricos", "Engenharia", "Geografia Física", "Geologia", "Ciências Ambientais e Ecologia”, "Estudos Urbanos" e "Meteorologia e Ciências Atmosféricas".

Em cima desta base de dados aplicou-se uma análise bibliométrica: método de análise e mensuração da produção de conhecimento científico baseado em três leis empíricas: Lei de Produtividade de Autores, Lei de Dispersão de Periódicos e Lei de Frequência das Palavras (Lotka,1926; Bradford,1934 \& Zipf, 1949, como citado em Araújo, 2006, p. 12).

De acordo com Pimenta et al. (2017), a principal atividade na qual a bibliometria baseia-se é a análise de citações realizadas entre o texto citante e o trecho citado. Para Foresti (1989), esse método, além de proporcionar o desenvolvimento do conhecimento científico, possibilita o reconhecimento do escritor, contribuindo para a construção de novas fontes de informações e expondo a literatura existente.

Os dados foram baixados no formato de arquivo do Microsoft Office Excel 2019 e foram classificados em categorias e quantificados em gráficos que mostram o percentual de publicações por área de conhecimento; o número de publicações por país; o total de publicações por ano; o total de citação por artigo; a média de citações por ano; o total de publicações e de citações por autor; o percentual e número de publicações por revista científica.

\section{Resultados e Discussão}

De acordo com o levantamento realizado, a produção científica internacional sobre a temática do rompimento de barragens, no período compreendido entre os anos de 1980 e início de 2021, encontra-se publicada na rede mundial de computadores num total de 306 artigos, distribuídos em 2.844 páginas de 58 periódicos indexados na coleção principal da base Web of Science. Os estudos abrangem 20 categorias da referida base de dados, dentre as quais se destacam dez áreas de conhecimento com maior relevância na produção científica sobre o tema em tela. 
O percentual de publicações por área de conhecimento está representado no Gráfico 1, em que se observa os maiores volumes de produção nas áreas de Recursos Hídricos (81,0\%) e Engenharia Civil (58,8\%).

Gráfico 1: Percentual de publicações por área de conhecimento.

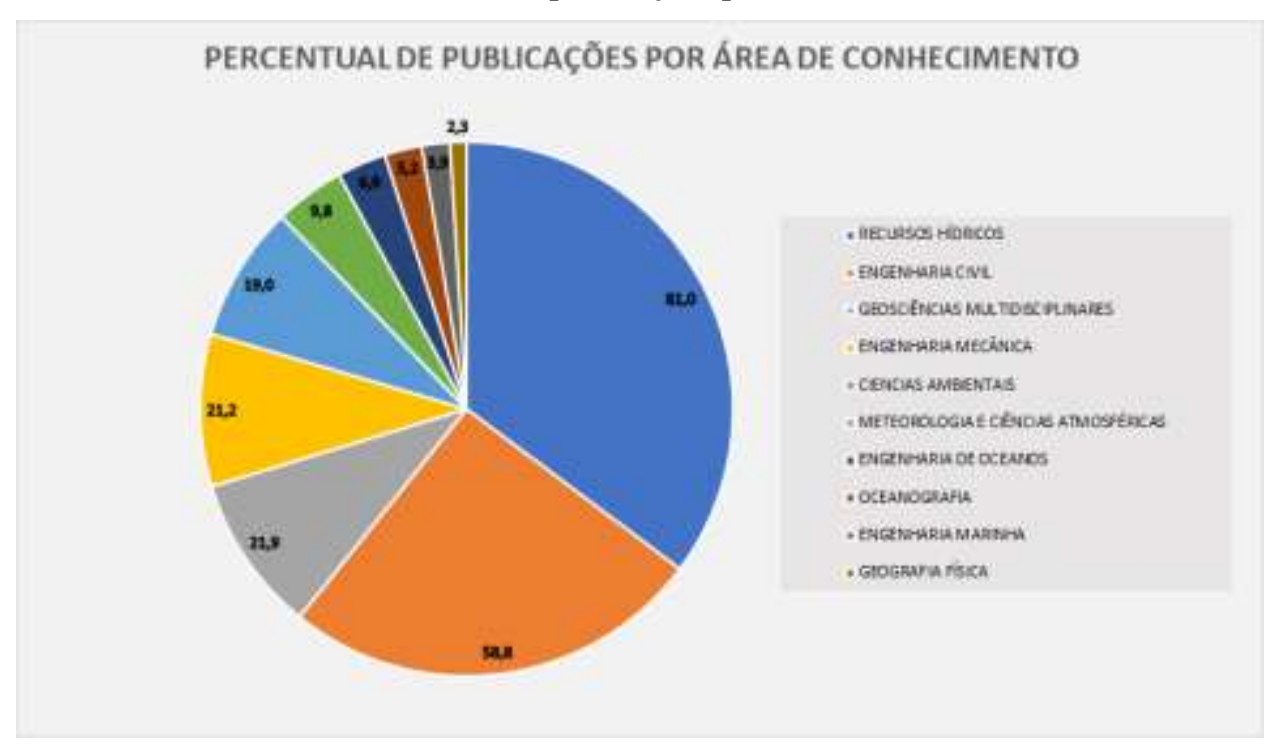

Fonte: Produzido a partir dos dados pesquisados na Web of Science, (2021).

O número total de artigos que abordam o rompimento de barragens, de acordo com os critérios de refinamento utilizados nesta pesquisa, está distribuído geograficamente por 25 países de 4 continentes, excetuando-se a África e a Antártida. (Figura 1) $)^{1}$.

Liderando o ranking de publicações está a República Popular da China com 94 artigos publicados, seguida pelos Estados Unidos com 47 publicações e a Itália com 35. Reino Unido, Canadá e Bélgica completam a lista dos 6 países que mais publicaram sobre o tema analisado, com 25, 16 e 14 publicações respectivamente. O Brasil aparece como o único país da América do Sul a publicar sobre rompimento de barragens, com 5 artigos publicados no período.

\footnotetext{
${ }^{1} \mathrm{Na}$ legenda da Figura 1 não aparecem os países: Grécia, Indonésia, México, Cazaquistão e Holanda, apesar de ambos estarem representados no mapa. Estes países contam com 4 publicações cada um.
} 
Figura1: Distribuição do número de publicações por países.

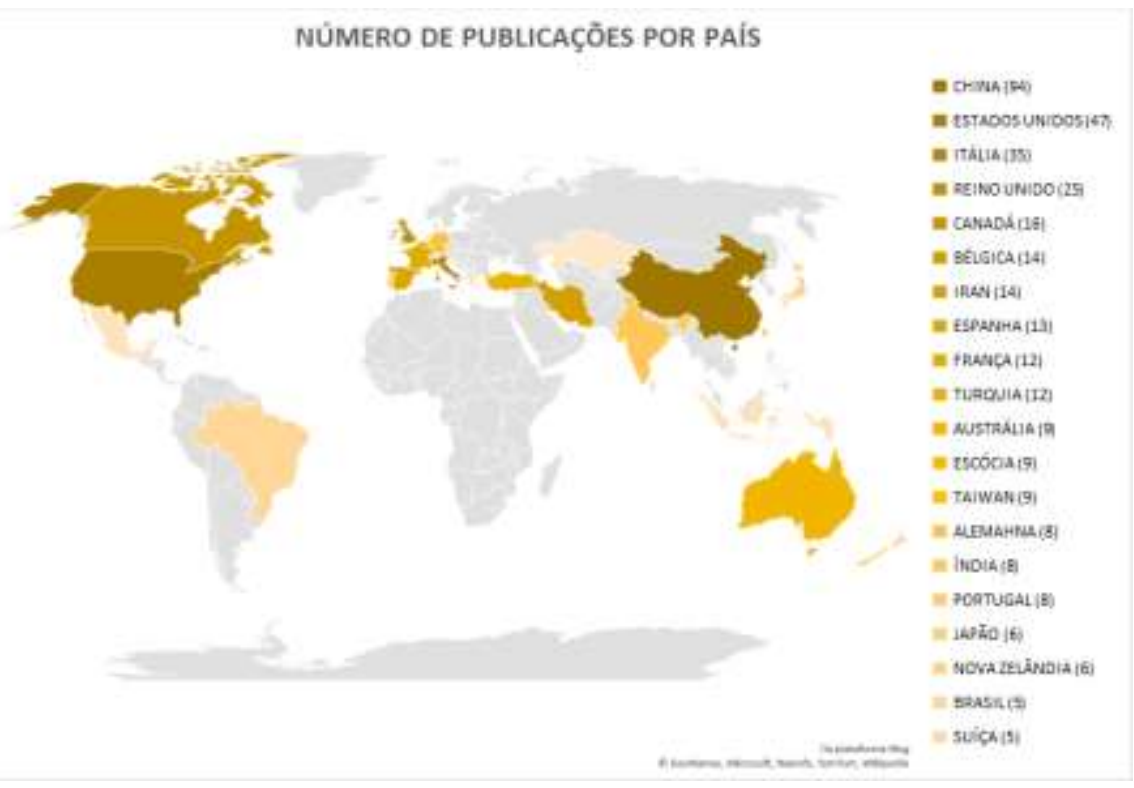

Fonte: Produzido no Microsoft Office Excel 2019 com dados da Base Web of Science 2021 plotados automaticamente na plataforma Bing, (2021).

A distribuição dos artigos por ano de publicação (Gráfico 2) mostra um significativo aumento no número de publicações nos últimos anos. Entre os anos de 2018 e 2020 registrou-se o maior aumento no número de publicações, com um crescimento de $206 \%$ entre estes dois anos. Estes dados mensuram a contribuição científica sobre o tema analisado ao longo do tempo, e revelam o aumento de sua relevância na literatura científica nos últimos anos.

Gráfico 2: Distribuição do total de artigos por ano de publicação.

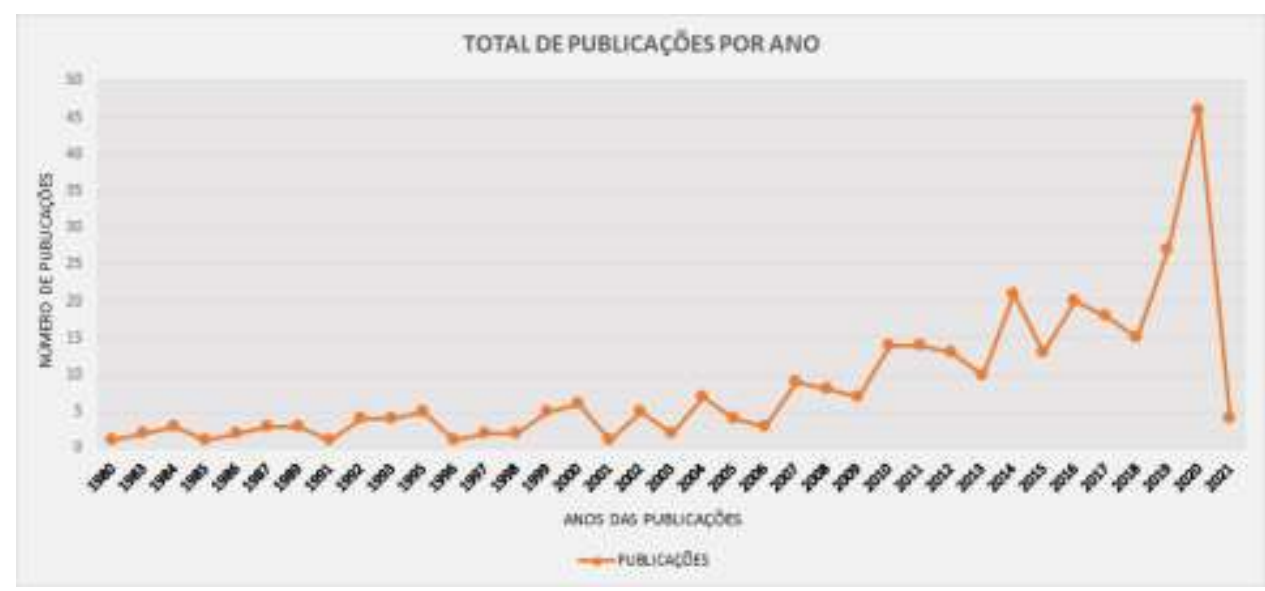

Fonte: Produzido a partir dos dados pesquisados na Web of Science, (2021).

A avaliação das publicações por nível de importância levou em consideração o H-index ordenado automaticamente pela Web of Science no ato da pesquisa. O H-index, proposto por Hirsch (2005), mensura o impacto das publicações de um autor de acordo com o número de suas publicações e o respectivo número de citações por outros autores. De acordo com Wanderley et al. (2014) o H-index é definido da seguinte forma: "um pesquisador possui um índice h se h dos seus Np artigos tem pelo menos h citações cada e os outros (Np-h) não tem mais do que h citações cada." Em outras palavras, um cientista tem um índice $\mathrm{h}=10$, se tiver 10 artigos publicados e cada um deles tiver sido citado pelo menos 10 vezes por outros trabalhos.

Do total dos 306 artigos pesquisados, 44 possuem maior impacto na literatura científica de acordo com o $\mathrm{H}$-index. Ou 
seja, esses artigos foram os mais citados em outros estudos e compõem a base de conhecimento mais sólida sobre o tema em tela.

Outros 37 artigos aparecem com zero citações, 22 deles publicados em 2020 e quatro em 2021. Talvez por se tratarem de publicações recentes, estes artigos ainda não foram citados em nenhum outro estudo.

Os 44 artigos de maior impacto foram ordenados de acordo com o total de citações recebidas, observando-se que oito deles, publicados entre os anos de 1995 e 2010, alcançaram mais de cem citações até então (Gráfico 3).

O artigo $n^{\circ} 1$ do ranking, publicado em 1995 no Journal of Hydraulic Research, Vol. 33, nº 6, sob a autoria de Luigi Fraccarollo e Eleuterio F. Toro, já conta com 238 citações.

Gráfico 3: Distribuição dos artigos de maior impacto pelo número de citações e seus respectivos anos de publicação.

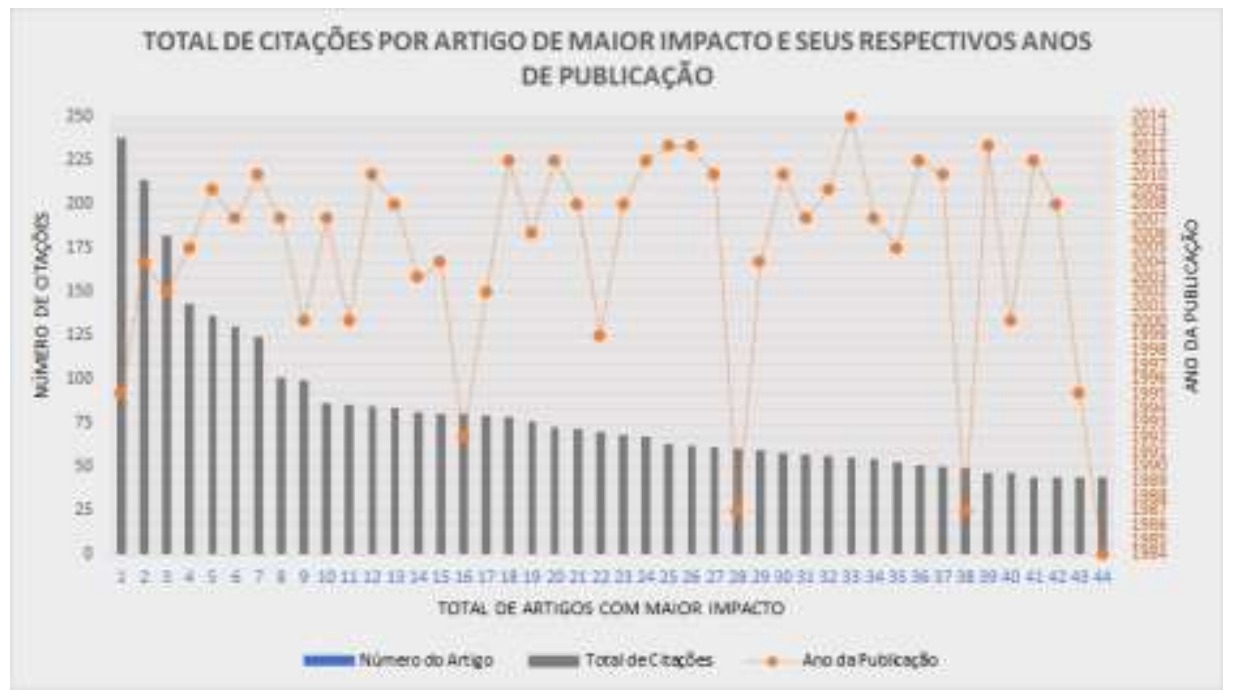

Fonte: Produzido a partir dos dados pesquisados na Web of Science, (2021).

A média de citações por ano dos oito artigos com mais de cem citações está representada no Gráfico 4 . $\mathrm{O}$ artigo $\mathrm{n}^{\circ} 2$ da sequência, de autoria de Cao, Z. et al (2004), apresentou a maior média de citações $(11,89)$, seguido do artigo $\mathrm{n}^{\circ}$ 5, de autoria de Gallegos, Humberto A.; Schubert, Jochen E. e Sanders, Brett F. (2009), com 10,46 de média.

A média de citações por ano leva em consideração o total de citações recebidas pelo artigo, dividido pelo número de anos contados a partir do ano da publicação do artigo. Por isso, o artigo $\mathrm{n}^{\mathrm{o}} 1$ - que tem um maior número de citações (238) tem uma média de citações por ano menor do que o artigo $\mathrm{n}^{\circ} 2$ - que só tem 214 citações. Isto acontece porque o artigo $\mathrm{n}^{\circ} 2$ (de 2004) é nove anos mais recente do que o artigo no 1 (de 1995). 
Gráfico 4: Média de citações por ano dos oito artigos de maior impacto com mais de cem citações.

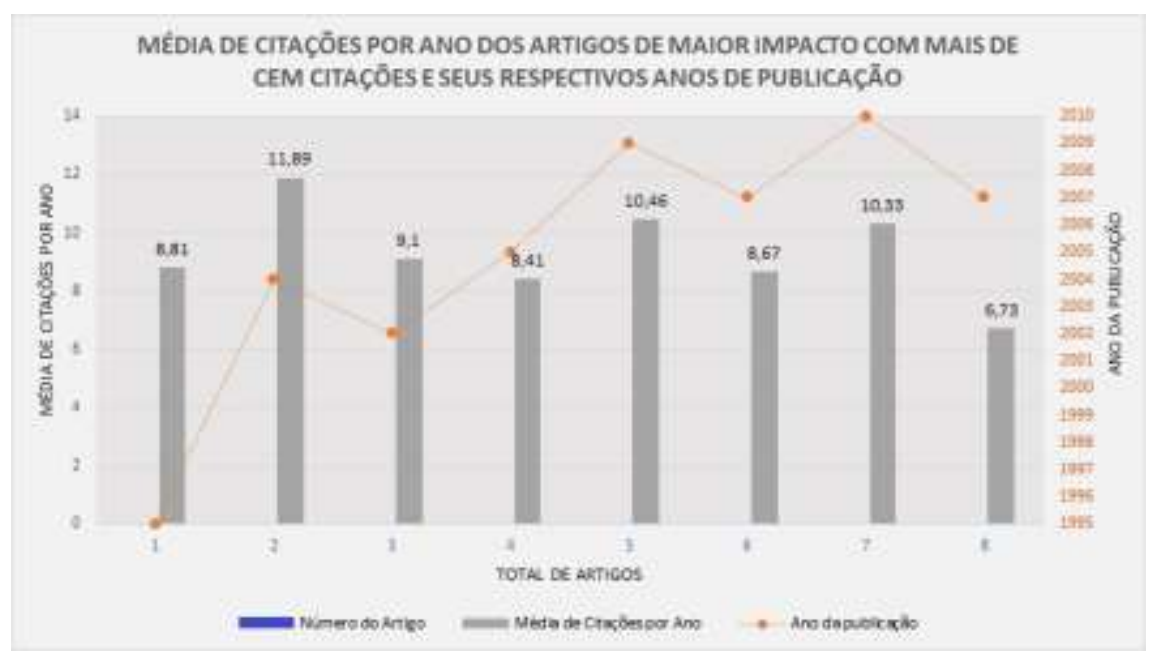

Fonte: Produzido a partir dos dados pesquisados na base Web of Science, (2021).

No tocante à relação de força por autor dentro da rede temática, este dado é obtido levando-se em consideração o número de coautoria por autor. Quanto maior o número de links de coautoria de um autor dentro da rede temática, maior será sua força dentro da rede. O Gráfico 5 mostra apenas os 42 autores - dentre os 700 que compõem a série pesquisada - que contam com cem ou mais citações dentro da rede temática analisada.

Os autores com maior número de documentos publicados e de citações recebidas dentro desta faixa são Zech, Y. e Soares-Frazão, S., com dez documentos publicados e total de citações de 509 e 407 respectivamente. A força destes dois autores dentro da rede temática é de 19 links, enquanto o autor Marazoni, A., com 5 documentos publicados, possui 153 citações e 20 links de coautoria.

Considerando toda a série dos 700 autores, o autor Chen, Y. é quem possui maior força dentro da rede temática analisada, com 42 links de coautoria.

Gráfico 5: Distribuição do número de documentos e de citações por autor e da relação de força por autor com mais de cem citações dentro da rede temática.

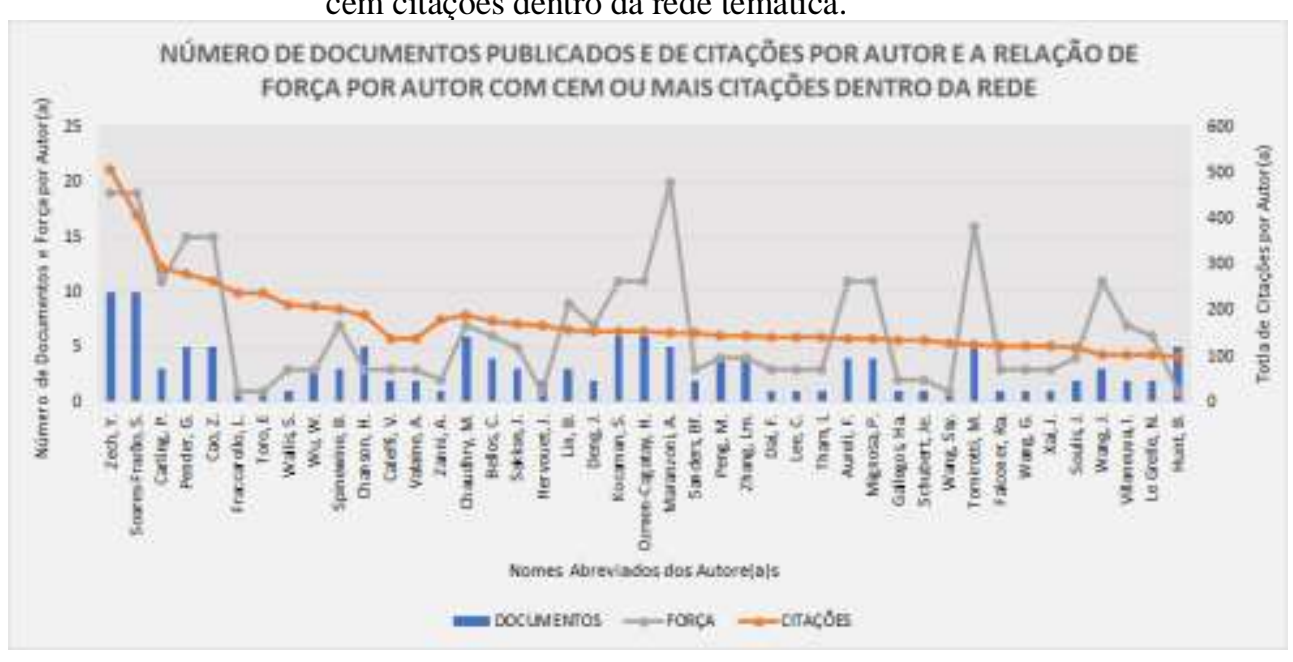

Fonte: Produzido a partir dos dados pesquisados na base Web of Science, (2021).

No tocante aos jornais, periódicos ou revistas científicas responsáveis pela publicação dos artigos pesquisados, analisou-se apenas os veículos mais importantes - aqueles que contam com mais de dez artigos publicados sobre o tema em tela. 
O Gráfico 6 mostra que o periódico mais importante para a temática do rompimento de barragens, com $16 \%$ das publicações, é o Journal of Hydraulic Research. Este periódico é o jornal principal da Associação Internacional de Engenharia e Pesquisa Hidroambiental (IAHR). Publica trabalhos de pesquisa em hidráulica teórica, experimental e computacional e mecânica dos fluidos, particularmente relacionados a rios, lagos, estuários, costas, hidrovias construídas e alguns fluxos internos, como fluxos de tubos. De acordo com a página do periódico na web (https://www.tandfonline.com/toc/tjhr20/current), o jornal conta com 100 mil downloads e visualizações anuais e tem um fator de impacto de 2.098 (ano de referência 2019). De acordo com a classificação de periódicos do quadriênio 2013-2016 disponível na Plataforma Sucupira (https://sucupira.capes.gov.br/sucupira/) em 2021, o Qualis do Journal of Hydraulic Research é A2 na área de avaliação Engenharias I.

Gráfico 6: Distribuição do número de artigos publicados por periódico com mais de dez publicações sobre rompimento de barragens e seus respectivos percentuais de publicação.

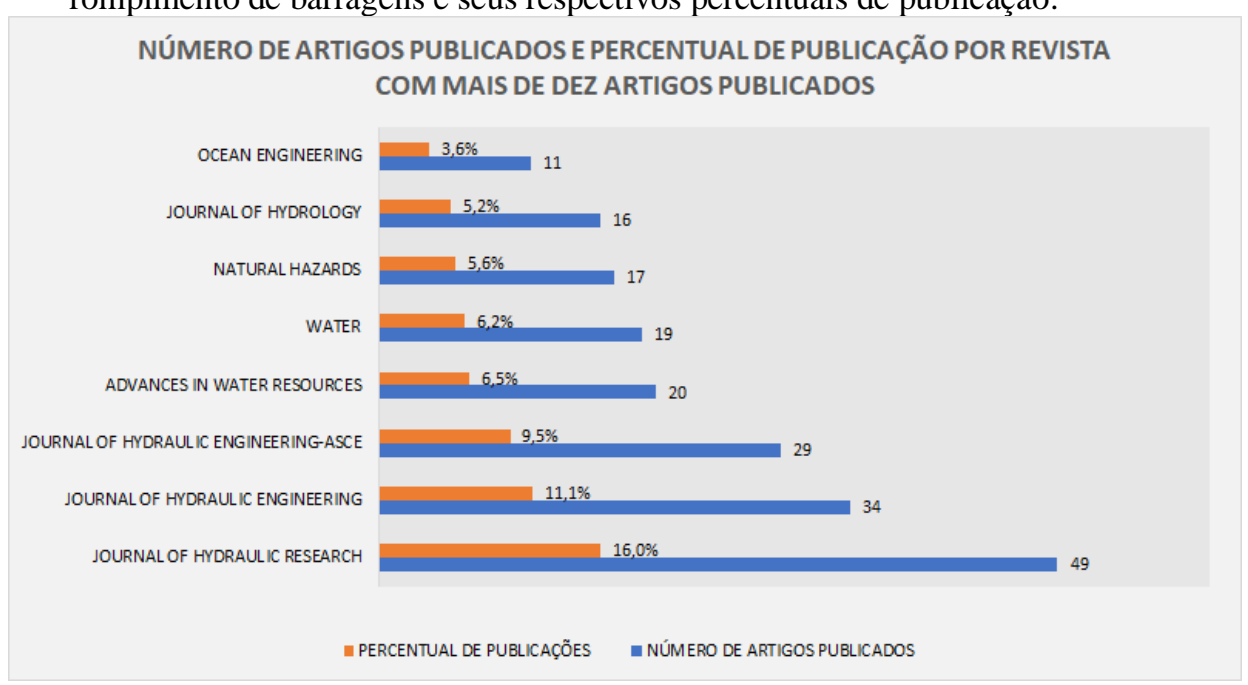

Fonte: Produzido a partir dos dados pesquisados na base Web of Science, (2021).

Em segundo lugar no ranking dos jornais que mais publicam sobre o tema aqui abordado está o Journal of Hydraulic Engineering, com 34 artigos e 11,1\% das publicações. O Jornal é publicado pela Sociedade Americana de Engenharia Civil e aceita contribuições originais que descrevem a análise e as soluções de problemas em engenharia hidráulica. A ênfase é colocada na apresentação de conceitos, métodos, técnicas e resultados que avançam o conhecimento e/ou são adequados para aplicação geral na profissão de engenharia hidráulica (SCImago, 2020). O Qualis do Journal of Hydraulic Engineering para o quadriênio 2013-2016, de acordo com a classificação de periódicos da Plataforma Sucupira (op. cit.) é A2 e B2 nas áreas de Engenharias III e IV respectivamente.

Por último, a Tabela 1 traz o ranking dos 44 artigos de maior impacto na produção científica sobre rompimento de barragens, de acordo com o H-index, descrevendo seus títulos, autores e anos de publicação para que possam ser conhecidos por pesquisadores interessados. 
Tabela 1: Ranking dos artigos de maior impacto segundo o H-index, seus títulos, autores e anos de publicação.

\begin{tabular}{|c|c|c|c|}
\hline $\mathbf{N}^{\mathbf{o}}$ & TÍTULO & AUTORES & ANO \\
\hline 1 & $\begin{array}{l}\text { Experimental and numerical assessment of the shallow water model for two- } \\
\text { dimensional dam-break type problems }\end{array}$ & Fraccarollo, L; Toro, EF & 1995 \\
\hline 2 & Computational dam-break hydraulics over erodible sediment bed & Cao, $\mathrm{Z}$ et al & 2004 \\
\hline 3 & $\begin{array}{l}\text { Case study: Malpasset dam-break simulation using a two-dimensional finite } \\
\text { volume method }\end{array}$ & $\begin{array}{l}\text { Valiani, A; Caleffi, V; } \\
\text { Zanni, A }\end{array}$ & 2002 \\
\hline 4 & $\begin{array}{l}\text { The } 1786 \text { earthquake-triggered landslide dam and subsequent dam-break flood } \\
\text { on the Dadu River, southwestern China }\end{array}$ & Dai, FC et al & 2005 \\
\hline 5 & $\begin{array}{l}\text { Two-dimensional, high-resolution modeling of urban dam-break flooding: A } \\
\text { case study of Baldwin Hills, California }\end{array}$ & $\begin{array}{l}\text { Gallegos, Humberto A.; } \\
\text { Schubert, Jochen E.; } \\
\text { Sanders, Brett F. }\end{array}$ & 2009 \\
\hline 6 & One-dimensional Modeling of dam-break flow over movable beds & $\begin{array}{l}\text { Wu, Weiming; Wang, } \\
\text { Sam S. Y. }\end{array}$ & 2007 \\
\hline 7 & Modelling dam-break flows over mobile beds using a 2D coupled approach & Xia, Junqiang et al & 2010 \\
\hline 8 & Small-scale laboratory dam-break waves on movable beds & Spinewine, B.; Zech, Y. & 2007 \\
\hline 9 & A high resolution 2-D dam-break model using parallelization & Hervouet, JM & 2000 \\
\hline 10 & Experimental study of dam-break flow against an isolated obstacle & $\begin{array}{l}\text { Soares-Frazao, S.; Zech, } \\
\text { Y. }\end{array}$ & 2007 \\
\hline 11 & Finite-difference TVD scheme for computation of dam-break problems & $\begin{array}{l}\text { Wang, JS; Ni, HG; He, } \\
\text { YS }\end{array}$ & 2000 \\
\hline 12 & CFD modelling approach for dam break flow studies & $\begin{array}{l}\text { Biscarini, C.; Di } \\
\text { Francesco, S.; Manciola, } \\
\text { P. }\end{array}$ & 2010 \\
\hline 13 & Modeling Dam Break Behavior over a Wet Bed by a SPH Technique & $\begin{array}{l}\text { Crespo, A. J. C.; Gomez- } \\
\text { Gesteira, M.; Dalrymple, } \\
\text { R. A. }\end{array}$ & 2008 \\
\hline 14 & Explicit schemes for dam-break simulations & Zoppou, C; Roberts, S & 2003 \\
\hline 15 & $\begin{array}{l}\text { Numerical prediction of dam-break flows in general geometries with complex } \\
\text { bed topography }\end{array}$ & Zhou, JG et al & 2004 \\
\hline 16 & Experimental Investigation of 2-Dimensional Dam-Break Induced Flows & $\begin{array}{l}\text { Bellos, CV; Soulis, JV; } \\
\text { Sakkas, JG }\end{array}$ & 1992 \\
\hline 17 & Dam break in channels with 90 degrees bend & $\begin{array}{l}\text { Soares Frazao, S; Zech, } \\
\text { Y }\end{array}$ & 2002 \\
\hline 18 & $\begin{array}{l}\text { Numerical simulation of shallow-water dam break flows in open channels using } \\
\text { smoothed particle hydrodynamics }\end{array}$ & Chang, Tsang-Jung et al & 2011 \\
\hline 19 & Tsunami surges on dry coastal plains: Application of dam break wave equations & Chanson, Hubert & 2006 \\
\hline 20 & A mesh-free particle model for simulation of mobile-bed dam break & $\begin{array}{l}\text { Shakibaeinia, Ahmad; } \\
\text { Jin, Yee-Chung }\end{array}$ & 2011 \\
\hline 21 & $\begin{array}{l}\text { Dam-break induced sediment movement: Experimental approaches and } \\
\text { numerical modelling }\end{array}$ & Zech, Y. et al & 2008 \\
\hline 22 & Malpasset dam-break revisited with two-dimensional computations & $\begin{array}{l}\text { Hervouet, JM; Petitjean, } \\
\text { A }\end{array}$ & 1999 \\
\hline 23 & Dam-break flow through an idealised city & $\begin{array}{l}\text { Soares-Frazao, Sandra; } \\
\text { Zech, Yves }\end{array}$ & 2008 \\
\hline 24 & A 2D shallow flow model for practical dam-break simulations & Wang, Yueling et al & 2011 \\
\hline 25 & $\begin{array}{l}\text { Analysis of human risks due to dam-break floods-part 1: a new model based on } \\
\text { Bayesian networks }\end{array}$ & Peng, M.; Zhang, L. M. & 2012 \\
\hline 26 & $\begin{array}{l}\text { Dam-break flows over mobile beds: experiments and benchmark tests for } \\
\text { numerical models }\end{array}$ & $\begin{array}{l}\text { Soares-Frazao, Sandra et } \\
\text { al }\end{array}$ & 2012 \\
\hline 27 & $\begin{array}{l}\text { Unsteady 1D and 2D hydraulic models with ice dam break for Quaternary } \\
\text { megaflood, Altai Mountains, southern Siberia }\end{array}$ & Carling, Paul et al & 2010 \\
\hline 28 & Simulation Of One-Dimensional Dam-Break Flows & $\begin{array}{l}\text { Fennema, RJ; Chaudhry, } \\
\text { MH }\end{array}$ & 1987 \\
\hline 29 & Numerical modeling of the initial stages of dam-break waves & $\begin{array}{l}\text { Shigematsu, T; Liu, PLF; } \\
\text { Oda, K }\end{array}$ & 2004 \\
\hline 30 & On particle-based simulation of a dam break over a wet bed & $\begin{array}{l}\text { Khayyer, Abbas; Gotoh, } \\
\text { Hitoshi }\end{array}$ & 2010 \\
\hline 31 & Experiments of dam-break wave over a triangular bottom sill & Soares-Frazao, S. & 2007 \\
\hline
\end{tabular}




\begin{tabular}{|c|c|c|c|}
\hline 32 & Application of the method of characteristics to the dam break wave problem & Chanson, Hubert & 2009 \\
\hline 33 & $\begin{array}{l}\text { 3-D finite-volume model of dam-break flow over uneven beds based on VOF } \\
\text { method }\end{array}$ & $\begin{array}{l}\text { Marsooli, Reza; Wu, } \\
\text { Weiming }\end{array}$ & 2014 \\
\hline 34 & $\begin{array}{l}\text { Large eddy simulation of stratified mixing in two-dimensional dam-break } \\
\text { problem in a rectangular enclosed domain }\end{array}$ & $\begin{array}{l}\text { Ozgokmen, Tamay M. et } \\
\text { al }\end{array}$ & 2007 \\
\hline 35 & $\begin{array}{l}\text { The October } 1999 \mathrm{Mt} \text { Adams rock avalanche and subsequent landslide dam- } \\
\text { break flood and effects in Poerua River, Westland, New Zealand }\end{array}$ & Hancox, GT et al & 2005 \\
\hline 36 & $\begin{array}{l}\text { Two-dimensional numerical modeling of dam-break flows over natural terrain } \\
\text { using a central explicit scheme }\end{array}$ & $\begin{array}{l}\text { Singh, Jaswant; } \\
\text { Altinakar, Mustafa S.; } \\
\text { Ding, Yan }\end{array}$ & 2011 \\
\hline 37 & Dam-break flows during initial stage using SWE and RANS approaches & $\begin{array}{l}\text { Ozmen-Cagatay, Hatice; } \\
\text { Kocaman, Selahattin }\end{array}$ & 2010 \\
\hline 38 & 1-D Dam-Break Flood-Wave Propagation On Dry Bed & Bellos, CV; Sakkas, JG & 1987 \\
\hline 39 & Initial stage of two-dimensional dam-break waves: laboratory versus VOF & $\begin{array}{l}\text { Oertel, Mario; Bung, } \\
\text { Daniel B. }\end{array}$ & 2012 \\
\hline 40 & $\begin{array}{l}\text { Numerical simulation and experimental verification of Dam-Break flows with } \\
\text { shocks }\end{array}$ & $\begin{array}{l}\text { Aureli, F; Mignosa, P; } \\
\text { Tomirotti, M }\end{array}$ & 2000 \\
\hline 41 & 1923 Gleno Dam Break: Case Study and Numerical Modeling & Pilotti, Marco et al & 2011 \\
\hline 42 & $\begin{array}{l}\text { Dam-break flows: Acquisition of experimental data through an imaging } \\
\text { technique and 2D numerical modeling }\end{array}$ & Aureli, Francesca et al & 2008 \\
\hline 43 & $\begin{array}{l}\text { First-Order And 2nd-Order Flux-Difference Splitting Schemes For Dam-Break } \\
\text { Problem }\end{array}$ & $\begin{array}{l}\text { JHA, AK; Akiyama, J; } \\
\text { Ura, M }\end{array}$ & 1995 \\
\hline 44 & Perturbation Solution for Dam-Break Floods & Hunt, B & 1984 \\
\hline
\end{tabular}

Fonte: Produzida a partir dos dados pesquisados na base Web of Science, (2021).

\section{Conclusão}

A temática de rompimento de barragens está representada na literatura científica internacional, publicada na rede mundial de computadores, por um total 306 artigos, dos quais 44 possuem maior impacto devido ao seu total de citações e relação de força de seus autores dentro da rede temática. A China é o país de origem da maioria das publicações sobre o tema (30,7\%). O tema é dominado pelas áreas de Recursos Hídricos (81\%) e Engenharia Civil (58,8\%).

Os periódicos que mais publicam sobre o tema são Journal of Hydraulic Research (16\%) e Journal of Hydraulic Enginnering (11,1\%). Os autores mais produtivos e mais citados são Zech, Y. e Soares-Frazão, S. com dez artigos cada um, e 509 e 407 citações, respectivamente. O autor mais influente - aquele com maior relação de força dentro da rede temática com mais de cem citações - é Marazoni, A., com 20 links de coautoria. Considerando toda a série dos 700 autores, o autor Chen, Y. é quem possui maior força dentro da rede temática analisada, com 42 links de coautoria.

Estes resultados sugerem que o tema pesquisado está em evidência nas áreas de Recursos Hídricos e Engenharia Civil, e tem crescido em importância acadêmica de acordo com o aumento do número de publicações registradas em todo o mundo ao longo do tempo.

A dinâmica bibliométrica de um tema acadêmico-científico revela-se pelas características de sua evolução nas dimensões espacial e temporal. A distribuição das publicações sobre o tema rompimento de barragens, da forma como foi revelada neste estudo, mostrou uma concentração espacial do número de publicações em países como a China, Estados Unidos e Itália. Este dado pode levar pesquisadores do tema a direcionarem a atenção para estes países a fim de aprimorarem seus estudos. Já em termos temporais, a distribuição das publicações se iniciou no ano de 1980 e registrou crescimento mais expressivo a partir do ano de 2001. Esta dinâmica evolutiva mostra uma tendência clara do aumento da relevância temporal do tema, expresso pelo contínuo progresso das referências ao mesmo em trabalhos científicos com o passar dos anos.

Além da dimensão espaço-temporal, a dinâmica bibliométrica do tema é expressa também na verificação da produção individual e em rede de seus autores e seus principais meios de divulgação. Este trabalho verificou a distribuição dos principais artigos e seus autores por ano de publicação, por meio de divulgação (periódico científico), e por links de coautoria entre os autores (relação de força dos autores dentro da rede temática). Este método possibilita ao pesquisador do tema identificar as 
principais referências no assunto e direcionar seu foco para as fontes de maior relevância científica.

A mensuração de todos esses dados oferece ao pesquisador do tema um relatório sintético sobre onde e quando estão concentradas as principais bibliografias relativas ao rompimento de barragens para subsidiar novos estudos e aprimorar o conhecimento e a contribuição científica no desenvolvimento de técnicas de segurança e de prevenção de acidentes com barragens.

\section{Referências}

Agência Nacional de Águas. (2016). Encarte especial sobre a bacia do Rio Doce: rompimento da barragem em Mariana/MG. [Versão digital em Adobe Reader]. https://arquivos.ana.gov.br/RioDoce/EncarteRioDoce_22_03_2016v2.pdf

Araújo, C. A. (2006). Bibliometria: evolução histórica e questões atuais. Em questão, 12(1), 11-32. https://www.seer.ufrgs.br/EmQuestao/article/view/16

Castro, L. S. \& Almeida, E. S. (2019). Desastres e desempenho econômico: avaliação do impacto do rompimento da barragem de Mariana. Geosul, 34(70), 406-429. doi: https://doi.org/10.5007/2177-5230.2019v34n70p406

Ferla, R. (2018). Metodologia simplificada para análise de aspectos hidráulicos em rompimento de barragens. (Dissertação de Mestrado, Instituto de Pesquisas Hidráulicas, Universidade Federal do Rio Grande do Sul). https://www.lume.ufrgs.br/handle/10183/180112

Foresti, N. (1989). Estudo da contribuição das revistas brasileiras de biblioteconomia e ciência da informação enquanto fonte de referência para a pesquisa (Dissertação de Mestrado, Departamento de Biblioteconomia da Universidade de Brasília). https://repositorio.unb.br/handle/10482/35501

Fundação Oswaldo Cruz (2019). Avaliação dos impactos sobre a saúde do desastre da mineração da Vale (Brumadinho, MG). [Nota técnica formato digital]. https://www.icict.fiocruz.br/sites/www.icict.fiocruz.br/files/Relat_Brumadinho_impacto_Saude_01022019.pdf

Hirsch, J. (2005) An index to quantify an individual's scientific research output. Proceedings of the National Academy of Sciences of the United States of America, 102(46), 16569-16572. doi: https://doi.org/10.1073/pnas.0507655102

International Comission on Large Dams. (2018). World Register of Dams. General Synthesis. [Portal] https://www.icoldcigb.org/GB/world_register/general_synthesis.asp

Laschefski, K. A. (2020). Rompimento de barragens em Mariana e Brumadinho (MG): desastres como meio de acumulação por despossessão. Ambientes: Revista de Geografia e Ecologia Política, 2(1), 98. http://e-revista.unioeste.br/index.php/ambientes/article/view/23299

Lei $n^{\circ} 12.334$, de 20 de setembro de 2010. Estabelece a Política Nacional de Segurança de Barragens destinadas à acumulação de água para quaisquer usos, à disposição final ou temporária de rejeitos e à acumulação de resíduos industriais, cria o Sistema Nacional de Informações sobre Segurança de Barragens e altera a redação do art. 35 da Lei $\mathrm{n}^{\circ}$ 9.433, de 8 de janeiro de 1997, e do art. $4^{\circ}$ da Lei $\mathrm{n}^{\circ} 9.984$, de 17 de julho de 2000 . Brasília. 2010. http://www.planalto.gov.br/ccivil_03/_ato2007-2010/2010/lei/112334.htm

Neves-Silva, P., \& Heller, L. (2020). Rompimento da barragem em Brumadinho e o acesso à água das comunidades atingidas: um caso de direitos humanos. Ciência e Cultura, 72(2), 47-50. doi: http://dx.doi.org/10.21800/2317-66602020000200013

Pimenta, A. A.; Portela, A. R. M. R.; Oliveira, C. B. \& Ribeiro, R. M. (2017). A bibliometria nas pesquisas acadêmicas. Scientia, 4(7), 1-13. http://flucianofeijao.com.br/novo/wp-content/uploads/2017/12/EDUCAR_PARA_A_CIDADANIA_FINANCEIRA.pdf

Proske, D. (2018). Comparison of dam failure frequencies and failure probabilities. Beton-und Stahlbetonbau, 113, 2-6. doi: https://doi.org/10.1002/best.201800047

Sánchez, L. H. (2013). Avaliação de Impacto Ambiental: conceitos e métodos (2a. ed.) São Paulo: Oficina de Textos.

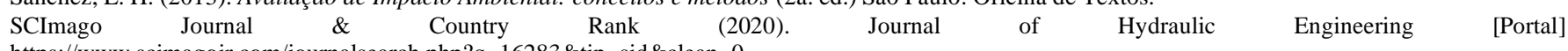
https://www.scimagojr.com/journalsearch.php?q=16283\&tip=sid\&clean=0

Spragens, L. C. \& Mayfield, S. M. (2005, 10 de maio). In safe hands: a profile of the ASDSO. International water power \& dam construction. https://www.waterpowermagazine.com/features/featurein-safe-hands-a-profile-of-the-asdso/

Torquetti, Z. S. C. \& Farias, R. S. (2004). Barragens industriais em MG: classificação quanto ao potencial de dano. Brasil Mineral. (232), 32-38

Tschiedel, A. F., Tassinari, L. C. S., Fan, F. M., \& Paiva, R. C. D. (2019). Barragens e rompimentos: compilação histórica nacional e internacional. In. Anais do XXIII Simpósio Brasileiro de Recursos Hídricos. (p. 10). Foz do Iguaçu, PR. Brasil: ABRHidro.

Universidade de Brasília - Biblioteca Central. (2018, julho, 12). Conheça a Web of Science. [Portal] Brasília: https://bce.unb.br/2018/06/conheca-a-web-ofscience/

Wanderley, A. J.; Duarte, A. N.; de Brito, A. V.; Prestes, M. A. \& Fragoso, F. C. (2014). Identificando correlações entre métricas de Análise de Redes Sociais e o h-index de pesquisadores de Ciência da Computação. In. Anais do III Brazilian Workshop on Social Network Analysis and Mining (pp. 45-56). Porto Alegre, RS. Brasil: SBC. 